\title{
III-V on Silicon DFB Laser Arrays
}

D. Van Thourhout, Y. Shi, B. Tian, Z. Wang

Photonics Research Group, INTEC

Ghent University, Ghent, Belgium dries.vanthourhout@ugent.be

Abstract - We will present our work on epitaxially grown III$\mathrm{V}$ on silicon DFB laser arrays, including results of pure InPbased lasers emitting around $900 \mathrm{~nm}$ and InGaAs-on-InP lasers emitting around $1300 \mathrm{~nm}$.
M. Pantouvaki, C. Merckling, B. Kunert, W. Guo, J. Van Campenhout

IMEC, Leuven, Belgium 